\title{
Tránsito al límite de la ciudad. Por los campos acotados de ruido entre el sujeto y la colectividad*
}

\author{
Josep Manuel Berenguer Alarcón \\ Orquesta del Caos \\ jmberenguer@sonoscop.net
}

\begin{abstract}
Resumen
Exploración introspectiva de la percepción sonora, desde lo más íntimo hasta lo más público, centrada en la experiencia de la estratificación del paisaje sonoro, que se relaciona con posibles mecanismos de individualización de estructuras cerradas y autónomas, contribuyentes en su generación y cuya actividad podría llegar a justificar la consideración del paisaje sonoro y sus elementos aislados en términos de formas continuamente mutantes de resultas de los procesos evolutivos a los que sus fuentes se hallarían sujetas.
\end{abstract}

Palabras clave

Paisaje sonoro, clausura sónica, ruido, evolución.

Transit to the limit of the city. Through the limited fields of noise between the subject and the community

\begin{abstract}
Introspective exploration of the sound perception, from the most intimate to the most public, centered on the experience of the stratification of the soundscape, which is related to possible mechanisms of individualization of closed and autonomous structures, contributing in its generation and whose activity could arrive to justify the consideration of the soundscape and its isolated elements in terms of continuously mutant forms, as a result of the evolutionary processes to which its sources would be subject.
\end{abstract}

Keywords

Soundscape, sonic closure, noise, evolution.

Transitar para o limite da cidade. Através dos campos delimitados de ruído entre o sujeito e a comunidade

Resumo

Exploração introspectiva da percepção do som, do mais íntimo ao mais público, centrada na experiência da estratificação da paisagem sonora, relacionada a possíveis mecanismos de individualização de estruturas fechadas e autonomas, contribuindo para

* Recibido: 15 de abril de 2017 / Aceptado: 31 de mayo de 2017 
Josep Berenguer. Tránsito al límite de la ciudad. Por los campos acotados de ruido...

a sua geração e cujas atividades poderiam chegar para justificar a consideração da paisagem sonora e seus elementos isolados em termos de formas continuamente mutantes, como resultado dos processos evolutivos aos quais suas fontes estarão sujeitas.

Palavras-chave

Paisagem sonora, clausura sônica, ruído, evolução. 


\section{Dentro}

Mientras consigas percibir de la forma más pasiva y el paisaje te envuelva sin que permitas que ningún objeto en particular retenga tu atención, si el paisaje entra en ti sin que lo sientas, si su geometría te atraviesa sin poseerte, sin que interpretes; si, aun así, eres capaz de oír, de escuchar, te encontrarás ante el sonido de lo más íntimo; aquel que se desvanece en cuanto el mundo entra en ti de la mano de la escucha, de la mirada o de cualquier otra percepción exterior suficientemente intensa y atenta; suficientemente profunda. Dejarás de escucharlo siempre que se perfile la particularidad de los límites del mundo, se revelen sus formas, sus cumbres, sus valles, y cada objeto se instale como por arte de magia en su plano, es decir, a la profundidad relativa del campo acústico que le corresponde.

El más radical e íntimo sonido de la rarefacción se manifiesta, en mi caso, como un pitido agudo de altura invariable y nivel bajo, también constante. Pero si le concedo mayor atención, como si se tratara de uno más de los sonidos del mundo -los otros sonidos-, lo percibo, además, ácido, porque es coloreado y brilla igual que el de algunas pantallas de datos cuando están mal ajustadas. Es complejo; bastante más que una senoide única y roma aislada ahí arriba, como podría parecer a la escucha no demasiado atenta, que sólo detectaría lo más sobresaliente de esa señal: una banda de ruido muy estrecha y aguda, centrada en torno a los $15 \mathrm{kHz}$, cuyo brillo probablemente se deba a que ni la distribución temporal ni el color de los destellos son perfectamente constantes. Ese sonido no es el único componente perceptible; además, hay, mucho más leve y esquiva, de contenido e intensidad variable, como si procediera de un plano espacial lejano, una banda de ruido muy ancha que casi cualquiera convendría en asociar a la respiración del mundo de ahí fuera, ese ruido de fondo permanente que, en última instancia, Ilena el universo (Serres, 1995: 62) y puede que hasta el vacío más elemental. Sin embargo, cuando estoy seguro de que nada a mi alrededor sería capaz de causar esa sensación tan persistente y real, pero tan tenue, comprendo que viene de mí mismo: es parte de mí. Soy yo. Quizá lo más esencial y desconocido de mi existencia sea ese residuo, mi ruido de fondo; el ruido, a secas, que brota de cualquier cosa que observe en detalle. Por eso, me es muy difícil distinguirlo completamente del que procede de fuera, a menos que me llegue información por un canal distinto del que me proporciona esa emergencia esquiva. Quién no quiere percibirlo o se rehúsa a aceptar su existencia, está obligado a construir filtros y vivir dependiendo de ellos, lo que, a su vez, generará ruido en algún lugar. Los sonidos de la rarefacción tienden a coincidir con los del núcleo de la intimidad. Como lo describiera y divulgara John Cage (1969: 134) -que probablemente padecía de tinnitus (Berenguer, 2014), como yo- tras su famosa experiencia en la cámara anecoica de la Universidad de Harvard, puede acompañar a ese agudo un grave 
muy tenue, apenas perceptible y tampoco nada puro, que, en mi caso, flotando en la superficie de la banda ancha de ruido, ora emerge, ora se sumerge. No se le opone; no podría, ya que, al ruido blanco, que es su propio complemento, nada puede oponérsele, salvo él mismo. Por el contrario, es su contexto y su contingencia; navega en él y es intrínsecamente distinto. Es su medio y, además de que posee bordes, contrariamente a aquel, no parece ser generado en ninguna instancia del sistema nervioso. Ni siquiera necesariamente en las propias estructuras sensitivas del oído, aunque sí muy cerca. Resultaría para mí de la transmisión por vías no aéreas de cosas como el paso de la sangre por los vasos cercanos, contracciones musculares involuntarias o movimientos ocasionales, a veces tetánicos, en la cadena de huesecillos del oído medio. Ambos tipos de señal integran el paisaje sonoro más recóndito del sujeto y, en ausencia de otras señales que los enmascaren, jamás terminan desvaneciéndose completamente. A menos que desplace la atención sonora hacia afuera, para centrarla en algún punto sonoro del mundo, mientras no mire ni escuche ni oiga fuera de mí, ellos continuarán aquí dentro.

Ese continuo de comportamiento mucho menos banal de lo que me permitiría apreciar la habitual superficialidad de la escucha cotidiana, tan y cada vez más fragmentadora de la experiencia, es el sonido de lo más íntimo; el núcleo sónico más secreto de la soledad. Muy rara vez se manifiesta aislado, porque casi nunca estamos completamente solos. Por eso, la mayor parte de las veces se percibe mezclado con otros sonidos procedentes de ámbitos periféricos, cuya intensidad y distribución espectral son capaces de enmascararlo. El más próximo de esos ámbitos continúa siendo propio del sujeto, pero la percepción de sus sonidos es mucho más dependiente del entorno que la de los anteriores. Quizá sea porque tales sonidos ya no estimulan directamente el sistema nervioso o las estructuras internas del oído, sino que emplean los canales por los que llegan los demás: la vía aérea, que pone en movimiento el tímpano, y la vía ósea, la cual, aunque implicando al tímpano, compromete preponderantemente los huesecillos, el fluido coclear y las estructuras que baña, que se ponen en movimiento a causa de la vibración de los huesos de la cabeza. Este nuevo fenómeno de flotación, de transición entre presencia y no presencia acústica de la intimidad del sujeto, se manifiesta en diversos escenarios habituales; como al realizar un esfuerzo psíquico, físico o en esas noches en que, incapaces de conciliar el sueño, nos hacemos conscientes de la imagen sonora del latir del corazón o de la respiración, tan variables según el ánimo y el estado de salud. También, como cuando percibimos, resultado de una digestión complicada, los sonidos del peristaltismo propio o, al realizar algún gesto, de preferencia brusco, los sonidos cortos y de apariencia percutiva de las articulaciones y de los huesos; al ocurrir, unos u otros se reparten momentáneamente el primer plano de la atención consciente y, de alguna forma, el yo 
se tiñe alternativamente de ellos. Se hace entonces difícil discernir entre las consciencias del yo y del mundo y la verdadera naturaleza de las entidades que se agazapan tras ella (Bateson, 1991: 303). Todo aquí parece asunto de memoria y de consciencia; acaso sólo de memoria, si es que la consciencia se deriva íntegramente de aquella.

\section{Ya afuera, pero no muy lejos}

En contacto íntimo con ese mundo sonoro interno, que, provisionalmente y en referencia al concepto de clausura operacional de Maturana y Varela (2003), Ilamaré clausura sónica. Ya claramente en el exterior, el sentido del oído alcanza el ámbito de los correlatos sonoros de la interacción con el entorno más próximo; aquel en virtud del cual el sujeto se relaciona por acoplamiento con todo lo que no forma parte de él, pero puede contribuir en la emergencia de sensaciones, así como también en su construcción, alterando sus estructuras o siendo, a su vez, transformado por su proximidad. Lo integran sonidos como el del roce de la ropa con el cuerpo o con sí misma, en función de los movimientos y de la naturaleza de la propia ropa; el de los pasos, que depende de las características físicas del calzado y del suelo, de su carácter e intencionalidad. Pero, además, está poblado por la hipercomplejidad sónica de nuestras herramientas, que casi son nosotros, ese ejército creciente de electrodomésticos, gadgets y robots que nos rodean, cuyas señales y epifenómenos sónicos se manifiestan imprevisibles, si los tomamos todos en conjunto. También, por la voz de las personas con quienes conversamos y nos relacionamos directamente; por los sonidos de los instrumentos que ellas emplean. Me referiré a este ámbito, también provisionalmente, como el borde externo de su clausura sónica y lo caracterizaré como el mundo sonoro de las acciones propias del sujeto y el de las de aquellos agentes de su entorno con capacidad de condicionarle y, a su vez, de ser condicionados por él. Por supuesto que el sonido se integra en los mecanismos de acoplamiento entre unos y otros, es decir, en las interfaces.

A partir de aquí, más allá del borde de la clausura sónica del oyente, se extiende un dominio sonoro estratificado de límites borrosos, acotables, pero no definibles, donde los planos sonoros sucesivos son percibidos cada vez con menor presencia; lejanía creciente, por supuesto, y valoración subjetiva de capacidad de afectación de nuestras realidades más inmediatas, generalmente decreciente. También, de sentimiento de atenuación de la influencia del oyente sobre las fuentes que representan y de forma directamente proporcional a la percepción de la distancia. La percepción resultante de ese conjunto sonoro, que se halla en movimiento constante e imprevisible, como las partículas de polen en suspensión en un líquido (Brown, 1866: 465-466), depende de una arena de relaciones espaciales de la que no es posible extraer umbrales absolutos que nos orienten en la 
resolución de la distancia a la que se halla una fuente sonora dada. En un conjunto complejo de sonidos más o menos distantes, la sensación de pertenencia de uno de ellos a un plano o a otro no tiene por qué mantenerse constante. Todo depende del tira y afloja de la lucha de sus intensidades por la consciencia, que varía a lo largo de su existencia y, así, influye en la aparición y desaparición de experiencias (Varela, Thomson y Rosch, 1997: 106). Es un casi-equilibrio de fuerzas contrastantes en el hecho de la máscara: la intensidad con la que la autonomía de cada ente sonoro se expresa y prevalece o no ante las otras y, en definitiva, resuelve la forma en que los entes sonoros se acoplan (Maturana y Varela, 2003: 121), en virtud de las propiedades funcionales de sus clausuras.

Con la distancia, la identificación de los sonidos aislados se torna cada vez más ardua para el sujeto; sus formas, sus perfiles, sus límites pierden definición, tienden a confundirse tanto con los de los sonidos que percibimos a su misma distancia, como con los de aquellos que pueblan los otros planos que el oído alcanza. De hecho, éste es un dominio de concurrencia por la atención consciente de todos los sonidos de todos los planos, porque los sonidos de los planos lejanos están expuestos a la contingencia de los cercanos, estadísticamente mucho más presentes y con capacidad muy superior de enmascaramiento de los límites de aquellos.

\section{Colectividad}

Los sonidos son, además, en cierta forma, residuos de la actividad de sus causantes. Productos de desecho, al tiempo que síntomas. Los que componen esa amalgama que mora más allá de la clausura sónica compiten por la atención consciente individual y también por el conjunto de las posibles atenciones conscientes pobladoras de un área dada. De ahí surge una idea que serviría para caracterizar lo urbano en términos de confluencia de interacciones sonoras entre clausuras operacionales humanas en condiciones de alta densidad, en detrimento de las producidas por fuentes sonoras no antropogénicas, en clara minoría, si se tiene en cuenta que el sonido producido por las máquinas también forma parte de los antropogénicos. La probabilidad de interacción y transformación de la autonomía de los moradores de las ciudades aumenta con la densidad. Con ella, la de los bordes externos de sus clausuras sónicas; así es que, desde esa perspectiva, el hecho de que la ciudad sea un lugar de conflicto acústico debería ser considerado como un fenómeno inherente a su naturaleza. El contexto urbano es un espacio donde el borde externo de la clausura sónica de cada sujeto se ve comprometida por la presencia de sus vecinos; otros sujetos, por cierto, de características atómicas comparables, lo que justificaría por sí solo una aproximación teórica basada en los presupuestos del caos determinista. Dada la supuesta contingencia de esa naturaleza, los conflictos sónicos urbanos no tendrían resolución posible. Solo les 
estaría dado evolucionar y, quizá, llegar a disolverse, pero esa situación nunca sería final; debería necesariamente dar paso a la generación de otros conflictos, tan caros a los entornos de alta densidad, que resulta atractivo tomarlos como una especie particularmente adaptada a ellos.

Lo enuncio sólo de paso y con mucha cautela: ¿y si la idea de superación de los conflictos guardara alguna relación con esa tan extrañamente extendida valoración positiva del crecimiento? Tal vez conviniera substituir los objetivos de superación de los conflictos con los de transición de un campo conflictivo a otro, a fin de que los sistemas se mantuvieran en movimiento constante, pero sin direccionalidad, de planicie en planicie, como supone Dawkins (1993: 100) que ocurre en la evolución de las especies. Así, la trascendencia de los conflictos tendría como resultado, no su evaporación, sino el cambio dinámico de los estados de ordenación de las cosas y, como epifenómeno, la transformación de las viejas formas culturales junto a la emergencia de las nuevas. Al fin y al cabo, si no existieran conflictos, estaríamos muertos. Nos importa, pues, más que volatilizar conflictos, el aprovechamiento de su energía de la manera más segura y constructiva posible. Sin más consideraciones que lo maticen, los conceptos de superación y de crecimiento son tan estáticos como los de decrecimiento o mantenimiento. Mientras no se demuestre lo contrario, su derivada es una constante, algo sin potencialidad alguna de cambio. Desde un punto de vista evolutivo, la derivada del movimiento browniano, el ruido blanco (Granero, 2011: 4), absolutamente imprevisible, resulta mucho más misterioso e inspirador que una simple y estéril recta.

\section{En el límite sonoro de la ciudad}

Siempre hemos estado expuestos a señales sonoras borrosas. Quizá el hecho diferencial urbano es que, en la ciudad, lo borroso de los sonidos da comienzo más cerca que en los contextos de baja densidad; como cuando nos rodea la niebla. Es habitual que los habitantes de las ciudades percibamos el campo acústico urbano desde su interior, pues casi cualquier punto de la ciudad se halla en su interior. La ciudad nos rodea casi todo el tiempo; así es que sus sonidos nos llegan de todas partes; como en la jungla, por cierto. En virtud del efecto de máscara que los unos ejercen sobre los otros, emergen y se sumergen rápidamente en ese magma sonoro rugiente y poco diferenciado que nos rodea al salir a la calle y en cuya forma global todos contribuyen en magnitud y forma poco determinables. Juntos, en esa dialéctica de inmersiones y emergencias fortuitas sólo aproximables por métodos inespecíficos, sorprendentemente cobran el mismo aspecto, aunque de mayor presión sonora, que la banda ancha de bajísimo nivel que percibo en condiciones de rarefacción del sonido, a solas, sin más motivo aparente que el daño acumulado en mi oído interno a lo largo de mi historia. 
Si desde el interior de la ciudad el paisaje sonoro lejano se percibe en todas direcciones, cabe preguntarse -o mejor, investigar-qué ocurre cuando viajamos a su confín, allí donde se relaciona con los municipios colindantes; si en el comportamiento del sonido se advierte alguna interacción o acoplamiento entre sus clausuras; si sus clausuras sónicas son intercambiables, complementarias, simbióticas, sintomáticas de algún aspecto específico o si no aportan información alguna; si el sonido varía a medida que nos desplazamos del centro al borde o si se producen cambios bruscos en la experiencia sonora al llegar cerca de él. Existen condicionantes de todo tipo que determinan ese espectro de posibilidades. Además, a pesar de lo similares que puedan ser las materias y las fuentes sonoras, su gramática parece muy distinta de una ciudad a otra. Es una cuestión de sucesión y superposición. De tiempo. Intervienen en ello, por ejemplo, la existencia o ausencia de campo abierto, de accidentes geográficos o urbanísticos entre las zonas habitadas de ambos municipios, de vías de comunicación transversales y/o longitudinales entre ambos territorios, así como de su profusión; la existencia o ausencia de planes de crecimiento de una u otra ciudad, de orografía del terreno y del uso real que las poblaciones hacen de todo lo anterior. También, las características culturales del empleo de los transportes; por ejemplo, la proporción entre los medios privados o públicos de transporte y su comportamiento a lo largo del día. Es una casuística muy compleja, que va tomando forma y se articula a lo largo de la historia de cada población y sus límites, que no son solo físicos, ya que poseen, además, dimensiones mentales y sociales. En cierta forma, la consciencia de los propios límites indica el grado de autoconocimiento de un ente. Los ciudadanos acostumbramos a no saber demasiado bien dónde termina y dónde empieza la ciudad. Puede que sea porque la mayoría de los puntos de la frontera entre dos ciudades es inalcanzable. No hay camino para llegar a todos.

La Orquestra del Caos (2012) escogió estas observaciones como punto de partida para la investigación que sustentó el proyecto de ZeppelinExpandit2012 y dio lugar a la creación de una base de datos consultable, Barcelona-So-Limit, compuesta de paisajes sonoros de veinte puntos situados en los límites de los distritos barceloneses con los municipios de la periferia de la ciudad y el mar: Ciutat Vella, Sants-Montjuïc, Les Corts, Sarrià-Sant Gervasi, Horta-Guinardó, Nou Barris, Sant Andreu y Sant Martí. Pensábamos, ya entonces, que muy pronto el paisaje sonoro de algunos límites de Barcelona cambiaría mucho. El de otros permanecería igual o similar durante un cierto tiempo. Seguro que a largo plazo cambiaría el de todos, de manera que en cualquier momento de cambio sería interesante y fructífero comparar las evoluciones que unos y otros paisajes sonoros habrían experimentado. Barcelona-So-Límit fue concebido con la intención de poner de manifiesto que el patrimonio surge de aspectos culturales sometidos a evolución, "memes", como los denominaría Dawkins (1993: 215). Cambia porque lo construimos día a 
día, como resultado de nuestras adaptaciones al medio, que incluye al propio paisaje sonoro en igual medida que el resto de producciones culturales. Esas manifestaciones puntuales podrían ser entendidas en términos de producciones metabólicas de los procesos culturales. Esto parece frustrante desde una perspectiva individual, por necesidad restringida a cortas duraciones, por ser rápidamente perecedera, pues, es que nuestras emisiones sonoras serán patrimonio cuando cambien suficiente como para que pierdan los atributos por los que en un momento dado son reconocidos. Para llegar a este punto de no retorno, habrá tenido que seguir una evolución y sus primeros generadores ya habrán desaparecido.

Generalmente, no somos conscientes de este tipo de cambios. Tendemos a actuar como si lo que nos rodea fuera a permanecer siempre inmutable. Sin embargo, todo varía y termina dejando de ser lo que es, para dar a veces paso a nuevas realidades. Los cambios en el paisaje sonoro son inevitables. No tiene sentido insistir en mantenerlo igual, como sugerirían las orientaciones nostálgicas. Sí lo tiene, por el contrario, el registro, que permite el análisis y alimenta la reflexión, la creación, el goce y cualquier otra cosa que se nos ocurra para contribuir en una evolución viable.

\section{Referencias}

Bateson, Gregory (1991). Pasos hacia una ecología de la mente. Ramón Alcalde, trad. Buenos Aires: Lohlé-Lumen. (Obra original publicada en 1972).)

Berenguer, Josep Manuel (2014). Tinnitus. En José Oliveras, curador. Drone. Paco Torres Monsó (catálogo de la exposición del 3 de octubre de 2014 al 18 de enero de 2015). Gerona: Bólit Centre d'Art Contemporani. 17-19. Recuperado el 20 de junio de 2017 de http://www.bolit.cat/download/712/3/libret-drone-eng.pdf.

Brown, Robert (1866). The miscellaneous botanical works of Robert Brown, 1. London: R. Hardwicke.

Cage, John (1969). A Year from Monday. Middletown: Wesleyan University Press.

Dawkins, Richard (1993). El gen egoísta. Las bases biológicas de nuestra conducta. Barcelona: Salvat. (Obra original publicada en 1974).

Granero, Rafael (2011, 22 de marzo). El método de las características aleatorias: Notas de clase. Homepage Rafael Granero Bélinchon. Université de Lyon. Recuperado el 20 de junio de 2017 de http://math.univ-lyon1.fr/ granero/files/edsedp.pdf.

Maturana, Humberto; Varela, Francisco (2003). El árbol del conocimiento: las bases biológicas del entendimiento humano. Buenos Aires: Lumen.

Orquestra del Caos. (2012). Barcelona-So-Limit (Zeppelin Expandit 2012). Barcelona: Sonoscop. Recuperado el 20 de junio de 2017 de http://sonoscop.net/bsl/. 
Josep Berenguer. Tránsito al límite de la ciudad. Por los campos acotados de ruido...

Serres, Michel (1998). Genesis. Geneviève James y James Nielson, trads. Ann Arbor: University of Michigan Press. (Obra original publicada en 1982).

Varela, Francisco; Thomson, Evan; Rosch, Eleanor (1997). De cuerpo presente. Ciencia cognitiva y experiencia humana. Carlos Gardini, trad. Barcelona: Gedisa. (Obra original publicada en 1991). 